\title{
Waist-to-height ratio, inflammation and CVD risk in obese children
}

Josune Olza ${ }^{1, *}$, Concepcion M Aguilera ${ }^{1}$, Mercedes Gil-Campos ${ }^{2}$, Rosaura Leis ${ }^{3}$, Gloria Bueno ${ }^{4}$, Miguel Valle ${ }^{5}$, Ramon Cañete ${ }^{2}$, Rafael Tojo ${ }^{3}$, Luis A Moreno ${ }^{6}$ and Angel Gil ${ }^{1}$

'Department of Biochemistry and Molecular Biology II, Institute of Nutrition and Food Technology, Centre for Biomedical Research (CIB), Lab 123, University of Granada, Avda. del Conocimiento s/n, 18016 Armilla, Granada, Spain: ${ }^{2}$ Paediatric Research and Metabolism Unit, Reina Sofía University Hospital, Maimonides Institute for Biomedical Research (IMIBIC) and Unit of Paediatric Endocrinology, Cordoba, Spain: ${ }^{3}$ Unit of Investigation in Nutrition, Growth and Human Development of Galicia, Paediatric Department, Clinic University Hospital of Santiago, University of Santiago de Compostela, Santiago de Compostela, Spain: ${ }^{4}$ Paediatric Department, Lozano Blesa University Hospital, University of Zaragoza, Zaragoza, Spain: ${ }^{5}$ Unit of Clinical Analyses, Valle de los Pedroches Hospital, Cordoba, Spain: ${ }^{6}$ GENUD (Growth, Exercise, Nutrition and Development) Research Group, Faculty of Health Sciences, University of Zaragoza, Zaragoza, Spain

Submitted 14 March 2013: Final revision received 7 November 2013: Accepted 8 November 2013: First published online 2 January 2014

\begin{abstract}
Objective: To evaluate the association between waist-to-height ratio (WHtR) and specific biomarkers of inflammation, CVD risk and endothelial dysfunction in prepubertal obese children.

Design: Prospective, multicentre case-control study matched by age and sex. Setting: Children were recruited between May 2007 and May 2010 from primarycare centres and schools in three cities in Spain (Cordoba, Santiago de Compostela and Zaragoza).

Subjects: Four hundred and forty-six (223 normal weight and 223 obese) Caucasian prepubertal children aged 6-12 years.

Results: WHtR was higher in the obese than in the normal-weight children. Blood pressure, waist circumference, weight, height, insulin, plasma lipids, leptin, resistin, abnormal neutrophil and monocyte counts, C-reactive protein, IL-6, IL-8, TNF- $\alpha$, myeloperoxidase, soluble intercellular adhesion molecule- 1 , selectin and plasminogen activator inhibitor-1 levels were higher in the obese than in the normal-weight group. Adiponectin and HDL-cholesterol were lower and glucose and metalloproteinase-9 showed no differences. Resistin, TNF- $\alpha$ and active plasminogen activator inhibitor-1 were associated with WHtR, a sensitive indicator of central obesity.

Conclusions: Our results lead to the hypothesis that changes in biomarker levels of insulin resistance, inflammation and CVD risk before puberty might induce metabolic consequences of obesity in obese children before reaching adulthood.
\end{abstract}

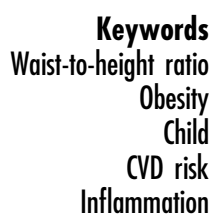

Obesity, especially excess visceral adiposity, is strongly associated with insulin resistance (IR), hypertension and dyslipidaemia, which contribute to high rates of morbidity and mortality ${ }^{(1)}$. Excess adiposity decreases the production of protective factors (e.g. adiponectin) and increases inflammatory molecules such as C-reactive protein $(\mathrm{CRP})^{(1)}, \mathrm{IL}_{-}{ }^{(1)}, \mathrm{TNF}^{\left({ }^{(2)}\right.}$ and resistin ${ }^{(3)}$, inducing a state of chronic low-grade inflammation ${ }^{(2,4)}$. Obese children have elevated levels of these inflammatory factors and pro-atherogenic biomarkers that are associated with CVD later in life ${ }^{(5)}$.

Obesity has been measured using a variety of anthropometric indices to estimate the risk for metabolic diseases.
Among the studied indices, the waist-to-height ratio (WHtR) is a strong predictor of CVD and diabetes ${ }^{(6)}$. WHtR was devised to correct for the over- and underestimation of risk among tall and short individuals with similar waist circumference ${ }^{(6)}$. This index is strongly correlated with abdominal fat measures which are obtained using advanced imaging techniques ${ }^{(7,8)}$. In a systematic review conducted in children, WHtR was associated with systolic and diastolic blood pressure (BP), high levels of insulin, HDL-cholesterol (HDL-C) and TAG in children and adolescents ${ }^{(6)}$. WHtR has also been associated with resistin levels in children and adolescents ${ }^{(9)}$, with plasminogen activator inhibitor-1 (PAI-1) levels in obese 
$\operatorname{adolescents}^{(10)}$ and with CRP levels in children and adolescents $^{(11-14)}$.

Abdominal adiposity leads to metabolic abnormalities and a low-grade inflammation including the production of adipokines, promotion of monocyte recruitment, differentiation of inflammatory monocytes into M1 phenotype macrophages and release of pro-inflammatory cytokines, such as CRP, TNF- $\alpha$ and IL-6, from adipose tissue ${ }^{(15)}$.

The large amount of evidence documenting the obesityrelated changes observed in children and adolescents, and the consequences of these changes in adulthood, has led to a search for biomarkers to enable early diagnosis. In the present study, we aimed to evaluate the association between adiposity (measured with the WHtR) and specific biomarkers of inflammation, CVD risk and endothelial dysfunction in a group of prepubertal obese children.

\section{Methods}

\section{Study design}

A case-control, multicentre study was performed in 446 prepubertal children paired by sex and age. The study was part of a SAMID Network (Research Network on Maternal and Child Health and Development), which is dedicated to the study of childhood health. The children included in the study were recruited from the centres within the network dedicated to the study of childhood obesity and its comorbidities. Childhood obesity was defined according to Cole $e t a l{ }^{(16)}$ using the age- and sex-specific cut-off points of BMI (corresponding to the adult cut-off of $30 \mathrm{~kg} / \mathrm{m}^{2}$ ). Inclusion criteria were prepubertal stage and absence of congenital metabolic diseases. Exclusion criteria were pubertal stage, the presence of infection, active or chronic inflammation, congenital metabolic diseases and the use of a medication that alters BP, glucose levels or lipid metabolism. After initial assessments at school or a primary-care centre, children who fulfilled the inclusion criteria were invited for a clinical examination in the appropriate participating hospital. The parents or guardians were informed about the purpose and procedures of the study before written consent was obtained, and all children gave their assent. The study was conducted according to the guidelines laid down in the Declaration of Helsinki (Edinburgh 2000 revised) and following the recommendations of Good Clinical Practice of the European Economic Community (document 111/3976/88, July 1990) and the legal in-force Spanish regulation that regulates Clinical Investigation in human beings (RD 223/04 about Clinical Assays). The study was approved by the Ethics Committee on Human Research of the University of Granada, the Ethics Committee of the Reina Sofía University Hospital of Cordoba, the Bioethics Committee of the University of Santiago de Compostela and the Ethics Committee in Clinical Research of Aragon.

\section{Study population}

We recruited 223 (119 males and 104 females) obese and 223 (119 males and 104 females) normal-weight children aged 6-12 years. All of the children were prepubertal and Caucasian and were recruited between May 2007 and May 2010 from primary-care centres and schools in three cities in Spain (Cordoba, Santiago de Compostela and Zaragoza). Children were classified according to Tanner stages by trained paediatricians and the prepubertal stage was confirmed by determination of sex hormone concentrations. The cut-off values for sex hormones were testosterone $<0.5 \mathrm{ng} / \mathrm{ml}$ in boys and oestradiol $<10 \mathrm{pg} / \mathrm{ml}$, luteinizing hormone $<5 \mathrm{U} / 1$ and follicle-stimulating hormone $<8 \mathrm{U} / 1$ in girls. Children with hormone levels higher than those mentioned were not included in the study.

\section{Antbropometric measurements}

Anthropometric measurements were taken by a single examiner at each hospital. Body weight was measured using a standard beam balance. Height was measured using a precision stadiometer. Waist circumference was measured in fasting state by applying an inelastic tape horizontally midway between the lowest rib margin and the iliac crest of the standing child at the end of a gentle expiration. BMI and WHtR were calculated. The $Z$-score for BMI (BMIZ) was based on reference standards published by Cole et $a l^{(17)}$.

\section{Blood pressure}

Systolic and diastolic BP were measured three times by the same examiner using a mercury sphygmomanometer and following international recommendations ${ }^{(18)}$. The mean of the three measurements was considered the current value.

\section{Biochemical analysis}

Blood samples were drawn from the antecubital vein between 08.00 and 09.30 hours after an overnight fast. The routine blood tests were analysed at the general laboratory of each hospital. The plasma aliquots used to measure inflammatory biomarkers were immediately frozen at $-80^{\circ} \mathrm{C}$ until analysed.

Glucose was analysed using the glucose oxidase method in an automatic analyser $(\mathrm{CV}=1 \cdot 0 \%$; RocheHitachi Modular P and D Autoanalyser; Roche Laboratory Systems, Mannheim, Germany) and plasma insulin was analysed by RIA ( $\mathrm{CV}=2 \cdot 6 \%)$ using an automatic microparticle analyser (AxSYM; Abbott Laboratories, Abbott Park, IL, USA). IR was calculated by the homeostatic model assessment of insulin resistance (HOMA-IR). Plasma TAG $(\mathrm{CV}=1 \cdot 5 \%)$, total cholesterol $(\mathrm{CV}=0.9 \%)$, HDL-C $(\mathrm{CV}=0 \cdot 8 \%)$ and LDL-cholesterol (LDL-C; $\mathrm{CV}=1 \cdot 5 \%)$ were measured using an automatic analyser (Roche-Hitachi Modular P and D Autoanalyser; Roche Laboratory Systems). 
Inflammatory, endotbelial dysfunction and CVD risk biomarkers

A complete blood count was performed in all children. The abnormal neutrophil count (AbnNC) was considered an inflammatory marker ${ }^{(19,20)}$. AbnNC levels greater than $6.6 \times 10^{3} \mu$ l were considered high ${ }^{(20)}$. Because monocytes are involved in the development of atheroma plaques, we used an abnormal monocyte count (AbnMC) of greater than $1 \cdot 02 \times 10^{3} \mu \mathrm{l}$, which represents the 95 th percentile of our normal-weight sample. Three different LINCOplex ${ }^{\mathrm{TM}}$ kits of human monoclonal antibodies (Linco Research, St Charles, MO, USA) were used in a Luminex ${ }^{\circledR} 200^{\mathrm{TM}}$ System (Luminex Corporation, Austin, TX, USA) to measure the biomarkers: (i) adiponectin $(\mathrm{CV}=7 \cdot 9 \%)$, resistin $(\mathrm{CV}=6 \cdot 0 \%)$ and active PAI-1 (aPAI-1; CV =6.6\%) (Cat. HADK1-61K-A); (ii) IL-6 (CV = 7·8\%), IL-8 (CV = 7·9\%), TNF- $\alpha(\mathrm{CV}=7 \cdot 8 \%)$ and leptin $(\mathrm{CV}=7 \cdot 9 \%)$ (Cat. HADK2$61 \mathrm{~K}-\mathrm{B}$ ); and (iii) myeloperoxidase (MPO; $\mathrm{CV}=12 \cdot 3 \%$ ), matrix metalloproteinase-9 (MMP-9; CV $=6 \cdot 8 \%$ ), total PAI-1 (tPAI-1; CV $=11 \cdot 2 \%$ ), soluble intercellular adhesion molecule-1 (sICAM-1; CV = 7.9\%) and soluble endothelial selectin (sE-selectin; $\mathrm{CV}=11 \cdot 2 \%$ ) (Cat. HCVD1-67 AK). CRP (CV = $4 \cdot 0 \%$ ) was measured using a particle-enhanced turbidimetric immunoassay (Dade Behring Inc., Deerfield, IL, USA).

\section{Statistical analysis}

Data were expressed as means with their standard errors. Normality was assessed with the Kolmogorov-Smirnoff test and the variables with a non-normal distribution were transformed. Homogeneity of variances was estimated using Levene's test. Mean comparisons between obese and normal-weight children for continuous variables were performed using the Student's $t$ test for unpaired samples. The $\chi^{2}$ test was applied to variables expressed as a percentage. The Pearson's test was applied to assess the relationship between WHtR and anthropometric, metabolic, inflammatory, CVD risk and endothelial dysfunction variables. Backward stepwise multiple linear regression analysis was performed to evaluate the independent risk factors associated with WHtR. Principal component factor analysis was used to determine if there was any particular grouping of biomarkers that were most related to WHtR in our study population. Extraction of the initial set of uncorrelated components was accomplished with the principal factor method and orthogonal rotation of components was then performed to facilitate interpretation. Seven variables related to WHtR were included in the factor analysis. Loadings were equivalent to Pearson correlation coefficients, with a higher loading indicating a stronger association between a factor and an observed variable. A factor loading from $0 \cdot 20$ to 0.39 was considered a marginal association, from 0.40 to 0.59 a moderate association, from 0.60 to 0.79 a strong association and above 0.80 a very strong association. Statistical significance was considered when $P<0 \cdot 05$. The statistical software package IBM SPSS Statistics 20 was used for all statistical analyses.

\section{Results}

\section{General, antbropometric and metabolic characteristics}

Table 1 shows the general and anthropometric characteristics of the children, confirming the similar distribution of age and sex between the normal-weight and obese groups. The mean weight, height, BMI, BMIZ, waist circumference and WHtR were all significantly higher in the obese group. Within the normal-weight group, there were no differences between sexes; however, within the obese group, boys had higher age, weight, height and BMIZ than girls.

In comparison to the normal-weight group, the obese group, both as a whole and stratified by sex, had higher systolic and diastolic BP, as well as higher plasma TAG, insulin and HOMA-IR levels. The obese group had lower HDL-C levels and the obese boys had lower total cholesterol. No differences were found in fasting glucose or LDL-C concentrations between the two groups (Table 1). Resistin and leptin levels were significantly higher in the obese group than in the normal-weight group, but adiponectin levels were lower. Within the normalweight group, only diastolic BP was higher in the girls and within the obese group, HDL-C was lower and insulin higher in the girls; the rest of the variables did not show differences.

\section{Biomarkers of inflammation, CVD risk and endotbelial dysfunction}

Table 2 shows the biomarkers of inflammation, CVD risk and endothelial dysfunction. In comparison to the normalweight group, the percentage of children with AbnNC, AbnMC as well as the levels of CRP, IL-6, IL- 8 and TNF- $\alpha$ were higher in the obese group. Similarly, biomarkers of CVD risk (MPO and total and active PAI-1) and endothelial dysfunction (sICAM-1 and sE-selectin) were significantly higher in the obese group compared with the normal-weight group. There was no difference in MMP-9 levels between groups. Within boys, all biomarkers except IL-6 and MMP-9 were higher in the obese group, and within the girls, all biomarkers were higher in the obese group except AbnNC, IL-6 and MMP-9. Within the obese group, only AbnNC in boys and aPAI-1 in girls were higher. Most of these biomarkers were associated with plasma TAG and HDL-C $(r=0 \cdot 106$ to $0 \cdot 332$; data not shown).

\section{Associations between waist-to-beight ratio and antbropometric measurements, insulin resistance and biomarkers of inflammation, CVD risk and endotbelial dysfunction}

Table 3 shows the associations with statistical significance between WHtR and the biomarkers of inflammation, endothelial dysfunction and CVD risk. After eliminating the multicollinearity (a situation in which two or more variables are very closely linearly related) using the IBM SPSS Statistics 20 software, a backward stepwise multiple 
Table 1 General, clinical and metabolic characteristics of the studied population: Caucasian prepubertal children aged 6-12 years from three cities in Spain (Cordoba, Santiago de Compostela and Zaragoza), May 2007-May 2010

\begin{tabular}{|c|c|c|c|c|c|c|c|c|c|c|c|c|c|c|c|c|c|}
\hline & \multicolumn{6}{|c|}{ Normal-weight } & \multicolumn{6}{|c|}{ Obese } & & & & & \\
\hline & \multicolumn{2}{|c|}{ All } & \multicolumn{2}{|c|}{ Male } & \multicolumn{2}{|c|}{ Female } & \multicolumn{2}{|c|}{ All } & \multicolumn{2}{|c|}{ Male } & \multicolumn{2}{|c|}{ Female } & \multicolumn{5}{|c|}{$P$ value } \\
\hline & Mean & SE & Mean & SE & Mean & SE & Mean & SE & Mean & SE & Mean & SE & * & $t$ & $\ddagger$ & $\S$ & $\|$ \\
\hline$n$ and \% & 223 & 100 & 119 & $53 \cdot 4$ & 104 & $46 \cdot 6$ & 223 & 100 & 119 & $53 \cdot 4$ & 104 & $46 \cdot 6$ & & & & & \\
\hline Sex $($ no. M/F) & \multicolumn{2}{|c|}{$119 / 104$} & \multicolumn{2}{|c|}{119} & \multicolumn{2}{|c|}{104} & \multicolumn{2}{|c|}{$119 / 104$} & \multicolumn{2}{|c|}{119} & \multicolumn{2}{|c|}{104} & & & & & \\
\hline Age (years) & $8 \cdot 96$ & $0 \cdot 10$ & $9 \cdot 12$ & $0 \cdot 10$ & $8 \cdot 78$ & $0 \cdot 10$ & $8 \cdot 88$ & $0 \cdot 10$ & $9 \cdot 15$ & $0 \cdot 10$ & $8 \cdot 57$ & $0 \cdot 20$ & 0.574 & 0.840 & 0.315 & 0.077 & 0.004 \\
\hline Weight (kg) & 28.96 & 0.37 & $29 \cdot 49$ & 0.51 & $28 \cdot 35$ & 0.55 & $52 \cdot 89$ & $0 \cdot 80$ & $55 \cdot 00$ & $1 \cdot 20$ & $50 \cdot 48$ & 0.97 & $<0.001$ & 0.001 & $<0.001$ & $0 \cdot 132$ & 0.004 \\
\hline Height $(\mathrm{m})$ & $1 \cdot 32$ & 0.01 & 1.33 & 0.01 & $1 \cdot 31$ & 0.01 & $1 \cdot 38$ & 0.01 & $1 \cdot 40$ & 0.01 & $1 \cdot 36$ & 0.01 & $<0.001$ & $<0.001$ & $<0.001$ & 0.073 & 0.001 \\
\hline BMI $\left(\mathrm{kg} / \mathrm{m}^{2}\right)$ & $16 \cdot 57$ & $0 \cdot 10$ & $16 \cdot 60$ & $0 \cdot 14$ & $16 \cdot 54$ & $0 \cdot 16$ & $27 \cdot 48$ & $0 \cdot 24$ & $27 \cdot 88$ & $0 \cdot 36$ & $27 \cdot 03$ & $0 \cdot 32$ & $<0.001$ & $<0.001$ & $<0.001$ & 0.785 & 0.085 \\
\hline BMIZ & -0.48 & $0 \cdot 13$ & $-0 \cdot 21$ & 0.05 & -0.22 & 0.05 & $3 \cdot 17$ & 0.04 & 4.03 & $0 \cdot 12$ & $3 \cdot 15$ & $0 \cdot 11$ & $<0.001$ & $<0.001$ & $<0.001$ & 0.886 & $<0.001$ \\
\hline WC $(\mathrm{cm})$ & $58 \cdot 48$ & 0.46 & $58 \cdot 55$ & 0.54 & $58 \cdot 41$ & 0.76 & $82 \cdot 82$ & 0.92 & $83 \cdot 81$ & $1 \cdot 30$ & $81 \cdot 72$ & $1 \cdot 29$ & $<0.001$ & $<0.001$ & $<0.001$ & 0.871 & 0.257 \\
\hline WHtR & 0.44 & 0.00 & 0.44 & 0.00 & 0.45 & 0.01 & $0 \cdot 60$ & 0.01 & 0.60 & 0.01 & 0.60 & 0.01 & $<0.001$ & $<0.001$ & $<0.001$ & 0.365 & 0.854 \\
\hline \multicolumn{18}{|l|}{ Clinical } \\
\hline Systolic BP (mmHg) & 96 & 1 & 95 & 1 & 97 & 1 & 109 & 1 & 109 & 1 & 110 & 1 & $<0.001$ & $<0.001$ & $<0.001$ & 0.252 & 0.501 \\
\hline Diastolic BP (mmHg) & 59 & 1 & 58 & 1 & 61 & 1 & 68 & 1 & 68 & 1 & 69 & 1 & $<0.001$ & $<0.001$ & $<0.001$ & $0 \cdot 017$ & 0.591 \\
\hline Glucose (mg/l) & 84 & 0 & 84 & 1 & 83 & 1 & 84 & 1 & 85 & 1 & 84 & 1 & 0.313 & 0.340 & 0.629 & 0.659 & 0.460 \\
\hline Insulin (mU/l) & $5 \cdot 18$ & $0 \cdot 18$ & 4.99 & $0 \cdot 20$ & $5 \cdot 41$ & $0 \cdot 31$ & $11 \cdot 21$ & 0.55 & $10 \cdot 38$ & 0.73 & $12 \cdot 21$ & $0 \cdot 81$ & $<0.001$ & $<0.001$ & $<0.001$ & 0.544 & 0.027 \\
\hline HOMA-IR & 1.09 & 0.04 & 1.04 & 0.04 & $1 \cdot 14$ & 0.07 & $2 \cdot 38$ & $0 \cdot 13$ & $2 \cdot 21$ & $0 \cdot 17$ & 2.58 & $0 \cdot 20$ & $<0.001$ & $<0.001$ & $<0.001$ & 0.601 & 0.050 \\
\hline \multicolumn{18}{|l|}{ Metabolic } \\
\hline Adiponectin (mg/l) & $26 \cdot 11$ & $0 \cdot 76$ & $25 \cdot 80$ & 0.96 & $26 \cdot 49$ & $1 \cdot 22$ & $21 \cdot 31$ & 0.75 & 21.95 & 1.04 & $20 \cdot 60$ & 1.08 & $<0.001$ & 0.001 & $<0.001$ & 0.872 & 0.645 \\
\hline Resistin ( $\mu \mathrm{g} / \mathrm{l})$ & $9 \cdot 27$ & $0 \cdot 28$ & $9 \cdot 32$ & 0.37 & 8.87 & 0.42 & $12 \cdot 04$ & 0.45 & 11.69 & 0.48 & $12 \cdot 36$ & 0.77 & $<0.001$ & $<0.001$ & $<0.001$ & 0.421 & 0.455 \\
\hline Leptin $(\mu \mathrm{g} / \mathrm{l})$ & $4 \cdot 05$ & $0 \cdot 26$ & $4 \cdot 21$ & $0 \cdot 40$ & $3 \cdot 87$ & $0 \cdot 31$ & $23 \cdot 33$ & 0.96 & 23.92 & $1 \cdot 38$ & $22 \cdot 67$ & $1 \cdot 34$ & $<0.001$ & $<0.001$ & $<0.001$ & 0.741 & 0.953 \\
\hline $\mathrm{TAG}(\mathrm{mg} / \mathrm{dl})$ & $53 \cdot 89$ & $1 \cdot 28$ & $51 \cdot 96$ & $1 \cdot 74$ & $56 \cdot 12$ & $1 \cdot 86$ & $77 \cdot 20$ & $2 \cdot 65$ & $74 \cdot 10$ & $3 \cdot 84$ & $80 \cdot 78$ & 3.59 & $<0.001$ & $<0.001$ & $<0.001$ & 0.072 & 0.058 \\
\hline $\mathrm{TC}(\mathrm{mg} / \mathrm{dl})$ & 172.55 & $2 \cdot 00$ & $173 \cdot 87$ & 2.99 & $171 \cdot 02$ & $2 \cdot 57$ & $164 \cdot 23$ & $2 \cdot 04$ & 163.69 & 3.07 & $164 \cdot 87$ & $2 \cdot 60$ & 0.004 & 0.018 & 0.094 & 0.477 & 0.774 \\
\hline HDL-C (mg/dl) & $65 \cdot 64$ & $1 \cdot 00$ & $66 \cdot 96$ & $1 \cdot 40$ & $64 \cdot 13$ & $1 \cdot 43$ & 51.69 & 0.89 & $53 \cdot 78$ & $1 \cdot 13$ & $49 \cdot 25$ & $1 \cdot 37$ & $<0.001$ & $<0.001$ & $<0.001$ & $0 \cdot 168$ & 0.007 \\
\hline LDL-C (mg/dl) & $94 \cdot 11$ & $1 \cdot 78$ & $93 \cdot 82$ & $2 \cdot 61$ & $94 \cdot 44$ & $2 \cdot 38$ & $96 \cdot 17$ & $1 \cdot 73$ & $94 \cdot 58$ & $2 \cdot 49$ & $98 \cdot 07$ & $2 \cdot 37$ & 0.408 & 0.835 & 0.282 & 0.862 & 0.316 \\
\hline
\end{tabular}

M, male; F, female; BMIZ, BMI Z-score; WC, waist circumference; WHtR, waist-to-height ratio; BP, blood pressure; HOMA-IR, homeostasis model assessment of insulin resistance; TC, total cholesterol; HDL-C, HDL-cholesterol:

To convert the values for glucose to $\mathrm{mmol} / /$, multiply by 0.055 ; to convert the values for insulin to pmol//, multiply by 6 ; to convert the values for TAG to mmol//, multiply by 0.01129 ; to convert the values for total cholesterol to $\mathrm{mmol} / \mathrm{l}$, multiply by 0.02586 .

Data are presented as means with their standard errors unless indicated otherwise. The $t$ test was used to compare between groups and sexes. Statistical significance was taken as $P<0.05$ for comparisons.

${ }^{*}$ All normal-weight $v$, all obese.

tNormal-weight males $v$. obese males.

sNormal-weight females $v$. obese females.

v. normal-weight females.

Obese males $v$. obese females. 
Table 2 Biomarkers of inflammation, CVD risk and endothelial dysfunction in the studied population: Caucasian prepubertal children aged 6-12 years from three cities in Spain (Cordoba, Santiago de Compostela and Zaragoza), May 2007-May 2010

\begin{tabular}{|c|c|c|c|c|c|c|c|c|c|c|c|c|c|c|c|c|c|}
\hline & \multicolumn{6}{|c|}{ Normal-weight } & \multicolumn{6}{|c|}{ Obese } & & & & & \\
\hline & \multicolumn{2}{|c|}{ All } & \multicolumn{2}{|c|}{ Male } & \multicolumn{2}{|c|}{ Female } & \multicolumn{2}{|c|}{ All } & \multicolumn{2}{|c|}{ Male } & \multicolumn{2}{|c|}{ Female } & \multicolumn{5}{|c|}{$P$ value } \\
\hline & Mean & SE & Mean & SE & Mean & SE & Mean & SE & Mean & SE & Mean & SE & * & $\dagger$ & $\ddagger$ & $\S$ & ॥ \\
\hline$n$ and \% & 223 & $100 \cdot 0$ & 119 & $53 \cdot 4$ & 104 & $46 \cdot 6$ & 223 & $100 \cdot 0$ & 119 & $53 \cdot 4$ & 119 & $53 \cdot 4$ & & & & & \\
\hline \multicolumn{18}{|l|}{ Inflammation } \\
\hline $\begin{array}{l}\text { Blood neutrophils }(\mathrm{U} / \mu \mathrm{l}) \\
\text { AbnNC (\%) }\end{array}$ & \multicolumn{2}{|c|}{1.9} & \multicolumn{2}{|c|}{$32120.0^{94}$} & \multicolumn{2}{|c|}{$\begin{array}{ll}3421 & 4 \cdot 0^{191}\end{array}$} & \multicolumn{2}{|c|}{$\begin{array}{ll}3876 & { }^{10} \\
6 \cdot 6\end{array}$} & \multicolumn{2}{|c|}{$3782{ }^{4 \cdot 8^{126}}$} & \multicolumn{2}{|c|}{$3983{ }^{3 \cdot 6} 6^{164}$} & $\begin{array}{l}<0.001 \\
<0.001\end{array}$ & $\begin{array}{r}<0.001 \\
0.022\end{array}$ & $\begin{array}{l}0.001 \\
0.192\end{array}$ & $\begin{array}{l}0.689 \\
0.028\end{array}$ & $\begin{array}{l}0.429 \\
0.276\end{array}$ \\
\hline $\begin{array}{l}\text { Blood monocytes }(U / \mu l) \\
\text { AbnMC (\%) }\end{array}$ & 4394.6 & 22 & 408 & 24 & $476 \quad 6 \cdot 1$ & 38 & $780_{21}$. & $7^{53}$ & 712 & 55 & 856 & 94 & $\begin{array}{l}<0.001 \\
<0.001\end{array}$ & $\begin{array}{l}<0.001 \\
<0.001\end{array}$ & $\begin{array}{l}<0.001 \\
<0.001\end{array}$ & $\begin{array}{l}0 \cdot 125 \\
0.357\end{array}$ & $\begin{array}{l}0.188 \\
0.333\end{array}$ \\
\hline CRP $(\mathrm{mg} / \mathrm{l})$ & 0.50 & 0.08 & 0.50 & $0 \cdot 12$ & 0.50 & $0 \cdot 10$ & $2 \cdot 45$ & $0 \cdot 19$ & $2 \cdot 17$ & 0.24 & 2.79 & $0 \cdot 31$ & $<0.001$ & $<0.001$ & $<0.001$ & 0.988 & $0 \cdot 107$ \\
\hline IL-6 (ng/l) & $4 \cdot 37$ & 0.55 & $4 \cdot 47$ & 0.75 & $4 \cdot 25$ & $0 \cdot 82$ & $7 \cdot 45$ & 0.89 & $6 \cdot 65$ & 1.07 & $8 \cdot 37$ & $1 \cdot 45$ & 0.003 & 0.097 & 0.015 & 0.842 & 0.333 \\
\hline IL-8 & $1 \cdot 58$ & $0 \cdot 11$ & $1 \cdot 61$ & $0 \cdot 18$ & $1 \cdot 53$ & $0 \cdot 11$ & $2 \cdot 29$ & $0 \cdot 18$ & $2 \cdot 28$ & $0 \cdot 26$ & $2 \cdot 31$ & 0.24 & 0.001 & 0.042 & 0.004 & 0.685 & 0.929 \\
\hline TNF- $\alpha(n g / l)$ & $3 \cdot 14$ & 0.11 & $3 \cdot 16$ & $0 \cdot 15$ & $3 \cdot 11$ & $0 \cdot 17$ & 3.91 & $0 \cdot 14$ & 4.06 & 0.20 & $3 \cdot 74$ & $0 \cdot 18$ & $<0.001$ & $<0.001$ & 0.008 & 0.776 & $0 \cdot 286$ \\
\hline \multicolumn{18}{|c|}{ CVD risk and endothelial dysfunction } \\
\hline MPO $(\mu \mathrm{g} / \mathrm{l})$ & $13 \cdot 63$ & $1 \cdot 27$ & $12 \cdot 72$ & $1 \cdot 72$ & $14 \cdot 67$ & $1 \cdot 8$ & $21 \cdot 97$ & $1 \cdot 83$ & $18 \cdot 79$ & $1 \cdot \varepsilon$ & $22 \cdot 04$ & $2 \cdot 18$ & $<0.001$ & $0 \cdot 017$ & 0.004 & 0.443 & 0.073 \\
\hline MMP. & $84 \cdot 88$ & $3 \cdot 33$ & $81 \cdot 11$ & 4.55 & $89 \cdot 25$ & $4 \cdot 89$ & $92 \cdot 10$ & $4 \cdot 69$ & $100 \cdot 28$ & $6 \cdot 9$ & $82 \cdot 73$ & $5 \cdot 60$ & 0.938 & $0 \cdot 119$ & 0.066 & 0.097 & 0.079 \\
\hline Active PAl-1 ( $\mu \mathrm{g} / \mathrm{l})$ & $5 \cdot 00$ & $0 \cdot 27$ & $4 \cdot 90$ & $0 \cdot 40$ & $5 \cdot 11$ & $0 \cdot 36$ & $13 \cdot 54$ & $0 \cdot 73$ & $11 \cdot 56$ & $0 \cdot 81$ & $15 \cdot 79$ & $1 \cdot 22$ & $<0.001$ & $<0.001$ & $<0.001$ & 0.340 & 0.007 \\
\hline Total PAI-1 & $18 \cdot 57$ & 0.84 & $18 \cdot 28$ & $1 \cdot 17$ & $18 \cdot 89$ & $1 \cdot 21$ & $28 \cdot 66$ & $1 \cdot 32$ & $26 \cdot 86$ & $1 \cdot 73$ & $30 \cdot 71$ & $2 \cdot 01$ & $<0.001$ & $<0.001$ & $<0.001$ & 0.382 & $0 \cdot 183$ \\
\hline & $0 \cdot 16$ & 0.00 & $0 \cdot 16$ & 0.01 & $0 \cdot 16$ & 0.01 & $0 \cdot 18$ & 0.01 & $0 \cdot 18$ & 0.01 & 0.18 & 0.01 & 0.001 & 0.012 & 0.020 & 0.909 & 0.925 \\
\hline $\mathrm{sE}$-selectin $(\mu \mathrm{g} / \mathrm{l})$ & $23 \cdot 27$ & 0.90 & $22 \cdot 93$ & 1.08 & $23 \cdot 65$ & $1 \cdot 41$ & $31 \cdot 50$ & $1 \cdot 13$ & $29 \cdot 90$ & $1 \cdot 49$ & $33 \cdot 34$ & $1 \cdot 70$ & $<0.001$ & $<0.001$ & $<0.001$ & 0.843 & $0 \cdot 110$ \\
\hline
\end{tabular}

AbnNC, abnormal neutrophil count (neutrophils were considered elevated when the AbnNC was $\left.>6.6 \times 10^{3} \mu\right)^{(8)}$; AbnMC, abnormal monocyte count (monocytes were considered elevated when the AbnMC was $>1.02 \times 10^{3} \mu \mathrm{l}$, representing the 95th percentile of the normal-weight population); CRP, C-reactive protein; MPO, myeloperoxidase; MMP-9, matrix metalloproteinase-9; PAI-1, plasminogen activator inhibitor-1; sICAM1 , soluble intercellular adhesion molecule-1; sE-selectin, soluble endothelial selectin.

Data are presented as means with their standard errors unless indicated otherwise. The $t$ test or the $\chi^{2}$ test (for percentages) was used to compare between groups and sexes. Statistical significance was taken as $P<0.05$ for comparisons.

${ }^{*}$ All normal-weight $v$. all obese.

tNormal-weight males $v$. obese males.

\#Normal-weight females $v$. obese females.

\$Normal-weight males $v$. normal-weight females.

IIObese males $v$. obese females. 
Table 3 Association of WHtR with biomarkers of metabolic dysfunction, inflammation, endothelial dysfunction and CVD risk in the studied population: Caucasian prepubertal children aged 6-12 years from three cities in Spain (Cordoba, Santiago de Compostela and Zaragoza), May 2007-May 2010

\begin{tabular}{lrr}
\hline Biomarker & $r$ & $P$ value \\
\hline Metabolic & & \\
Systolic BP & 0.408 & $<0.001$ \\
Diastolic BP & 0.353 & $<0.001$ \\
Insulin & 0.508 & $<0.001$ \\
HOMA-IR & 0.487 & $<0.001$ \\
TAG & 0.317 & $<0.001$ \\
HDL-C & -0.424 & $<0.001$ \\
Adiponectin & -0.211 & $<0.001$ \\
Resistin & 0.272 & $<0.001$ \\
Inflammation & & \\
Leucocytes & 0.162 & 0.002 \\
CRP & 0.325 & $<0.001$ \\
IL-6 & 0.110 & 0.030 \\
IL-8 & 0.130 & 0.008 \\
TNF- $\alpha$ & 0.215 & $<0.001$ \\
Endothelial dysfunction and CVD risk & & \\
sICAM-1 & 0.127 & 0.009 \\
sE-selectin & 0.250 & $<0.001$ \\
MPO & 0.109 & 0.030 \\
Active PAI-1 & 0.464 & $<0.001$ \\
Total PAl-1 & 0.295 & $<0.001$ \\
\hline
\end{tabular}

WHtR, waist-to-height ratio; BP, blood pressure; HOMA-IR, homeostasis model assessment of insulin resistance; HDL-C, HDL-cholesterol; CRP C-reactive protein; sICAM-1, soluble intercellular adhesion molecule-1, sE-selectin, soluble endothelial selectin; MPO, myeloperoxidase; PAI-1, plasminogen activator inhibitor-1.

Table 4 Final model of the backward stepwise regression analysis of WHtR with biomarkers of metabolic dysfunction, inflammation and CVD risk in the studied population: Caucasian prepubertal children aged 6-12 years from three cities in Spain (Cordoba, Santiago de Compostela and Zaragoza), May 2007-May 2010

\begin{tabular}{lrcr}
\hline Biomarker & \multicolumn{1}{c}{$\beta$} & SE & $P$ value \\
\hline HOMA-IR & 0.266 & 0.008 & $<0.001$ \\
Systolic BP & 0.188 & 0.000 & $<0.001$ \\
HDL-C & -0.179 & 0.005 & $<0.001$ \\
Active PAI-1 & 0.174 & 0.012 & $<0.001$ \\
CRP & 0.129 & 0.002 & 0.003 \\
TNF- $\alpha$ & 0.111 & 0.009 & 0.008 \\
Resistin & 0.087 & 0.001 & 0.047 \\
\hline
\end{tabular}

WHtR, waist-to-height ratio; HOMA-IR, homeostasis model assessment of insulin resistance; BP, blood pressure; HDL-C, HDL-cholesterol; PAI-1, plasminogen activator inhibitor-1; CRP, C-reactive protein.

linear regression analysis of WHtR with associated risk factors showed that systolic BP, HOMA-IR, resistin, HDL$\mathrm{C}, \mathrm{CRP}, \mathrm{TNF}-\alpha$ and aPAI- 1 were independently associated with WHtR (Table 4).

The principal factor analysis shown in Table 5 demonstrates that two factors were sufficient to explain the association between the biomarkers and WHtR. These two well-defined factors were together CVD risk factor and IR (composed of aPAI-1, resistin, HOMA-IR, HDL-C and systolic BP) and inflammation (composed of TNF- $\alpha$ and CRP). These two factors explained $49 \cdot 06 \%$ of the total variance in the data; the first factor explained $29 \cdot 83 \%$ of the variance and the second factor explained $19 \cdot 25 \%$.
Table 5 Principal factor analysis of the risk factors and WHtR in the studied population: Caucasian prepubertal children aged 6-12 years from three cities in Spain (Cordoba, Santiago de Compostela and Zaragoza), May 2007-May 2010

\begin{tabular}{lcc}
\hline Variable & CVD risk and IR & Inflammation \\
\hline Loading factor $^{*}$ & & \\
Active PAI-1 & 0.735 & \\
Resistin & 0.643 & -0.396 \\
HOMA-IR & 0.707 & \\
HDL-C & -0.648 & 0.327 \\
Systolic BP & 0.531 & 0.773 \\
TNF- $\alpha$ & 0.277 & 0.653 \\
CRP & 31.90 & 19.20 \\
Percentage & 31.90 & 51.10 \\
Variance & & \\
Cumulative variance & & \\
\hline
\end{tabular}

WHtR, waist-to-height ratio; IR, insulin resistance; PAl-1, plasminogen activator inhibitor-1; HOMA-IR, homeostasis model assessment of insulin resistance; HDL-C, HDL-cholesterol; $\mathrm{BP}$, blood pressure; $\mathrm{CRP}$, C-reactive protein.

*Loading factor is the linear association between an observed variable and an underlying factor. Relative loadings: very strong $(>0.80)$, strong $(0.60$ to $0.79)$, moderate $(0.40$ to 0.59$)$ and marginal $(0.20$ to 0.39$)$.

\section{Discussion}

Our study shows that there are differences between normal-weight and obese prepubertal children regarding biomarkers of inflammation, endothelial dysfunction and CVD risk. Specifically, we have demonstrated the association of WHtR, which is a sensitive measure of visceral adiposity, with resistin, TNF- $\alpha$ and aPAI- 1 levels in a population of prepubertal obese children.

The present study also yielded results that were in agreement with previous publications that showed an association between WHtR and common clinical and metabolic markers. In this regard, the most studied parameters are systolic and diastolic $\mathrm{BP}^{(21-23)}$, HOMA-IR and insulin ${ }^{(23,24)}$ TAG $^{(23,24)}$ and HDL-C ${ }^{(21-23)}$; all of them have been associated with WHtR in children and adolescents. However, beyond that, we studied specific biomarkers of inflammation, endothelial dysfunction and CVD risk, and when observing our results we wondered about the biological relevance of the differences of these biomarkers in obese children compared with normal-weight children. Unfortunately, there are no well-established cut-off values for these biomarkers in prepubertal children, and transferring reference values from adolescents or adults could pose a risk because there are many hormonal changes occurring during and after puberty that may affect their plasma values (e.g. adiponectin) ${ }^{(25)}$. Notwithstanding, cut-off values for some lipid biomarkers, namely TAG, HDL-C and LDL-C, are well known in childhood ${ }^{(26,27)}$ and in the present study most of our obese children had altered plasma levels of those parameters. Moreover, most of the inflammatory, CVD risk and endothelial dysfunction biomarkers were closely associated with plasma lipids whose significance in terms of biological relevance has been validated in both children and adults, supporting the results of the new associated biomarkers in prepubertal children. 
CRP, a well-known biomarker of inflammation, has been associated with adiposity in children and adolescents $^{(28)}$ and with measures of adiposity, including WHtR, in overweight German adolescent girls ${ }^{(14)}$, Australian adolescents ${ }^{(11)}$, Hispanic and non-Hispanic black children and adolescents ${ }^{(12)}$ and Chilean girls younger than 4 years ${ }^{(13)}$. In the present study, we found that CRP was associated with adiposity in an exclusive population of prepubertal children and independently associated with WHtR.

An interesting finding from the present study is the association of WHtR with resistin. Studies of obese, non-diabetic subjects have shown higher resistin levels compared with normal-weight subjects and direct associations between resistin levels and BMI. Using computed tomography imaging, other groups have more closely associated resistin with quantitative levels of visceral and subcutaneous fat and with abdominal and intrathoracic fat depots ${ }^{(3)}$. Additionally, several studies have demonstrated the effect of resistin on vascular cell function. Resistin increases the expression of the adhesion molecules vascular cell adhesion molecule- 1 and ICAM-1, promotes endothelial cell activation ${ }^{(3)}$, stimulates the macrophage secretion of pro-inflammatory cytokines and participates in glucose homeostasis and insulin sensitivity ${ }^{(29)}$. Li et al. $^{(9)}$ demonstrated the association between resistin and WHtR in a mixed population of children and adolescents; similarly, in the present study we demonstrated this association but in a population of prepubertal children and, additionally, an independent association between both measures. Furthermore, it was interesting to note that resistin was also associated with HOMA-IR and sE-selectin but not with sICAM-1 (data not shown), suggesting that IR at young ages leads to endothelial damage. However, more evidence is needed to draw conclusions in this population.

aPAI-1, another biomarker of CVD risk that contributes to the inflammation and IR present in obesity, has been associated with visceral fat in adults ${ }^{(30)}$, adolescents and children $^{(31)}$. aPAI-1 has been shown to be independent of insulin and TAG levels, total fat mass, insulin sensitivity and age $^{(32)}$. However, despite all the information regarding PAI-1 and its relationship with obesity, few authors have studied the association between this biomarker and metabolic and clinical factors or other specific biomarkers of inflammation, CVD risk and endothelial dysfunction in obese, prepubertal children ${ }^{(10)}$. For this reason, we performed an additional analysis and found that PAI-1 was associated not only with WHtR and with all the components of the metabolic syndrome, but also with CRP, leucocyte count, adiponectin, resistin, leptin, MMP-9, sE-selectin and sICAM-1 levels (see online supplementary material, eTable 1). Overall, these results suggest that children with a high WHtR might develop CVD at early ages because they already have altered risk biomarkers for this group of diseases. However, this conclusion should be taken with caution as biomarker concentrations change during growth and particularly puberty.

In our study, we also found that plasma TNF- $\alpha$, which is an inflammatory cytokine that has a crucial role in obesity-related IR, was independently associated with WHtR. To the best of our knowledge, this finding has been reported previously in adults ${ }^{(33)}$, but not in children.

Based on the principal factor analysis, we observed two well-defined factors that are associated with WHtR, (i) IR-CVD risk and (ii) inflammation, which together explain more than $50 \%$ of the variability in WHtR. The importance of the variables included in these two factors is also supported by the multiple linear regression analysis. Previously published findings along with those of the present study suggest that obesity and associated changes related to IR, inflammation and CVD risk cause systemic metabolic consequences in prepubertal children that compromise their health, leading them to develop from an early age diseases that, until recent years, were characteristic of adulthood.

\section{Strengths and limitations of the study}

The strengths of the present study are the sample size of a very-well defined group of prepubertal children and the large number of biomarkers assessed, compared with other previously reported studies ${ }^{(11-14)}$. Moreover, relationships of WHtR, a validated index of adiposity, with specific biomarkers of inflammation, CVD risk and endothelial dysfunction found in the current study are another major strength. On the other hand, the limitations are the lack of cut-off values of these biomarkers for children at prepubertal age to validate the biological relevance and the size of our sample to establish these values.

\section{Conclusions}

In summary, resistin, TNF- $\alpha$ and aPAI- 1 , which are biomarkers of IR, inflammation and CVD risk, respectively, are associated with WHtR in prepubertal obese children. Our results lead to the hypothesis that changes of biomarker levels before puberty might induce metabolic consequences of obesity in obese children before reaching adulthood.

\section{Acknowledgements}

Sources of funding: The study was supported by the Junta de Andalucía (Excellence Project CTS-2203) and the Instituto de Salud Carlos III and the Ministerio de Ciencia e Innovación (Red SAMID-RETIC n.RD08/0072). The Junta de Andalucía, Instituto de Salud Carlos III and Ministerio de Ciencia e Innovación had no role in the design, analysis or writing of this article. Conflicts of interest: All authors declare that they do not have any conflicts of interest. Authors' contributions: J.O. carried out the biomarker assays, participated in the statistical 
analysis, analysis and interpretation of the data, wrote the draft of the manuscript and participated in the critical revision of the manuscript. C.M.A. and L.A.M. participated in the design of the study and the critical revision of the manuscript. M.G.-C., R.L., G.B., M.V., R.T. and R.C. participated in the acquisition of the data. A.G. participated in the design and conception of the study, in the statistical analysis, analysis and interpretation of the data and in the critical revision of the manuscript. All authors read and approved the final manuscript.

\section{Supplementary material}

To view supplementary material for this article, please visit http://dx.doi.org/10.1017/S1368980013003285

\section{References}

1. Ouchi N, Parker JL, Lugus JJ et al. (2011) Adipokines in inflammation and metabolic disease. Nat Rev Immunol 11, 85-97.

2. Tzanavari T, Giannogonas P \& Karalis KP (2010) TNF- $\alpha$ and obesity. Curr Dir Autoimmun 11, 145-156.

3. Schwartz DR \& Lazar MA (2011) Human resistin found in translation from mouse to man. Trends Endocrinol Metab 22, 259-265.

4. Weyer C, Yudkin JS, Stehouwer CD et al. (2002) Humoral markers of inflammation and endothelial dysfunction in relation to adiposity and in vivo insulin action in Pima Indians. Atherosclerosis 161, 233-242.

5. Lloyd LJ, Langley-Evans SC \& McMullen S (2010) Childhood obesity and adult cardiovascular disease risk: a systematic review. Int J Obes (Lond) 34, 18-28.

6. Browning LM, Hsieh SD \& Ashwell M (2010) A systematic review of waist-to-height ratio as a screening tool for the prediction of cardiovascular disease and diabetes: 0.5 could be a suitable global boundary value. Nutr Res Rev 23, 247-269.

7. Soto González A, Bellido D, Buño MM et al. (2007) Predictors of the metabolic syndrome and correlation with computed axial tomography. Nutrition 23, 36-45.

8. Ashwell M, Cole TJ \& Dixon AK (1996) Ratio of waist circumference to height is strong predictor of intraabdominal fat. BMJ 313, 559-560.

9. Li M, Fisette A, Zhao XY et al. (2009) Serum resistin correlates with central obesity but weakly with insulin resistance in Chinese children and adolescents. Int J Obes (Lond) 33, 424-439.

10. Mantovani RM, Rios DR, Moura LC et al. (2011) Childhood obesity: evidence of an association between plasminogen activator inhibitor-1 levels and visceral adiposity. $J$ Pediatr Endocrinol Metab 24, 361-367.

11. Huang RC, de Klerk N, Mori TA et al. (2011) Differential relationships between anthropometry measures and cardiovascular risk factors in boys and girls. Int J Pediatr Obes $\mathbf{6}$, e271-e282.

12. Cossio S, Messiah SE, Garibay-Nieto N et al. (2009) How do different indices of obesity correlate with cardiometabolic disease risk factors in multiethnic youths? Endocr Pract 15, 403-409.

13. Corvalan C, Uauy R, Kain J et al. (2010) Obesity indicators and cardiometabolic status in 4-y-old children. Am J Clin Nutr 91, 166-174.

14. Kleiser C, Schienkiewitz A, Schaffrath Rosario A et al (2011) Indicators of overweight and cardiovascular disease risk factors among 11- to 17-year-old boys and girls in Germany. Obes Facts 4, 379-385.
15. Brooks GC, Blaha MJ \& Blumenthal RS (2010) Relation of C-reactive protein to abdominal adiposity. Am J Cardiol 106, 56-61.

16. Cole TJ, Bellizzi MC, Flegal KM et al. (2000) Establishing a standard definition for child overweight and obesity worldwide: international survey. BMJ 320, 1240-1243.

17. Cole TJ, Freeman JV \& Preece MA (1990) Body mass index reference curves for the UK, 1990. Arch Dis Child 73, $25-29$.

18. McCrindle BW (2010) Assessment and management of hypertension in children and adolescent. Nat Rev Cardiol 7, $155-163$.

19. Madjid M, Awan I, Willerson JT et al. (2004) Leukocyte count and coronary heart disease: implications for risk assessment. J Am Coll Cardiol 44, 1945-1956.

20. Skinner AC, Steiner MJ, Henderson FW et al. (2010) Multiple markers of inflammation and weight status: cross-sectional analyses throughout childhood. Pediatrics 125, e801-e809.

21. Hara M, Saitou E, Iwata F et al. (2010) Waist-to-height ratio is the best predictor of cardiovascular disease risk factors in Japanese school children. J Atheroscler Thromb 9, 127-132.

22. Ruiz JR, Ortega FB, Loit HM et al. (2007) Body fat is associated with blood pressure in school-aged girls with low cardiorespiratory fitness: the European Youth Heart Study. J Hypertens 25, 2027-2034.

23. Botton J, Heude B, Kettaneh A et al. (2007) Cardiovascular risk factor levels and their relationships with overweight and fat distribution in children: the Fleurbaix Laventie Ville Santé II study. Metabolism 56, 614-622.

24. Freedman DS, Dietz WH, Srinivasan SR et al. (2009) Risk factors and adult body mass index among overweight children: the Bogalusa Heart Study. Pediatrics 123, 750-757.

25. DeBoer MD (2013) Obesity, systemic inflammation, and increased risk for cardiovascular disease and diabetes among adolescents: a need for screening tools to target interventions. Nutrition 29, 379-386.

26. Hickman TB, Briefel RR, Carroll MD et al. (1998) Distributions and trends of serum lipid levels among United States children and adolescents ages 4-19 years: data from the Third National Health and Nutrition Examination Survey. Prev Med 27, 879-890.

27. National Heart, Lung, and Blood Institute, National Cholesterol Education Program (1991) Report of the Expert Panel on Blood Cholesterol Levels in Children and Adolescents. Publication no. NIH91-2732. Bethesda, MD: National Institutes of Health.

28. Steene-Johannessen J, Kolle E, Reseland JE et al. (2010) Waist circumference is related to low-grade inflammation in youth. Int J Pediatr Obes 5, 313-319.

29. Stofkova A (2010) Resistin and visfatin: regulators of insulin sensitivity, inflammation and immunity. Endocr Regul $\mathbf{4 4}$, 25-36.

30. Cigolini M, Targher G, Bergamo Andreis IA et al. (1996) Visceral fat accumulation and its relation to plasma hemostatic factors in healthy men. Arterioscler Thromb Vasc Biol 16, 368-374.

31. Caballeros AE, Bousquet-Santos K, Robles-Osorio L et al. (2008) Overweight Latin children and adolescents have marked endothelial dysfunction and subclinical vascular inflammation in association with excess body fat and insulin resistance. Diabetes Care 31, 576-582.

32. Janand-Delenne B, Chagnaud C, Raccah D et al. (1998) Visceral fat as a main determinant of plasminogen activator inhibitor 1 level in women. Int J Obes Relat Metab Disord 22, 312-317.

33. Wu SH, Shu XO, Chow WH et al. (2013) Adiposity and fat distribution in relation to inflammation and oxidative stress in a relatively lean population of Chinese women. Dis Markers 34, 279-293. 